\section{Endoscopic full-thickness resection based on the novel Dortmund technique}

In 2010, we performed full-thickness resection (FTR) using the over-the-scope clip (OTSC) system (Ovesco Endoscopy GmbH, Tübingen, Germany) [1]. The key aspects of this technique are to apply the clip to the target lesion and then to resect above the ensnared lesion. However, these maneuvers require several separate accessories. Therefore, a new device to perform FTR was developed. Herein we describe the steps to perform FTR using this novel platform.

The first case was a 74-year-old patient with an adenocarcinoma (pT1G1sm1) of the sigmoid colon, which was found by chance after endoscopic polypectomy. On pathology there was evidence of incomplete resection (R1), and therefore surgery was mandated. The second case was a 74-year-old patient with an adenocarcinoma (pT1G1sm1) of the ascending colon after previous polypectomy and an insecure safety margin in the vertical (i.e. depth) margin. Although surgery was indicated for both patients, they preferred the alternative option of undergoing endoscopic FTR.

- Video 1 demonstrates the endoscopic procedure. First, the area of interest or lesion is targeted. Second, the target lesion is suctioned or pulled inside the transpar- ent distal cap using the Twin grasper (Ovesco). Third, the clip (14/6t) is released below the invaginated tissue, creating a pseudopolyp. The tight grasping of the tissue with the clip results in secure wall apposition, preventing any perforation. Subsequently, the endoscopic resection is performed by means of a polypectomy snare. The lesion is entrapped above the deployed clip and then resected using electrosurgical cautery (Erbe, Tübingen, Germany). The resected specimen is then suctioned into the cap, retrieved, and sent to pathology. In both patients an R0 was achieved. No further interventions were needed.

We believe that this new FTR platform allows for en bloc and R0 resection of selected gastrointestinal lesions, which otherwise may mandate an invasive surgical approach. Whereas the traditional OTSC system also permits the resection of smaller lesions, this new device has better deployment mechanism and an attached snare ("all-in-one" system), making it easier to handle.

\section{Video 1}

Demonstration of new "all-in-one" technique for full-thickness resection.
Competing interests: None

\section{Martin Faehndrich, Michael Heike, Marcel Sandmann}

Interventional Endoscopy, Department of Gastroenterology, Klinikum Dortmund, Dortmund, Germany

\section{Reference}

1 Sandmann M, Heike M, Faehndrich M. Application of the OTSC system for the closure of fistulas, anastomosal leakages and perforations within the gastrointestinal tract. Z Gastroenterol 2011; 49: 981 -985

\section{Bibliography}

DOI http://dx.doi.org/

10.1055/s-0034-1377436

Endoscopy 2014; 46: E424

(c) Georg Thieme Verlag KG

Stuttgart · New York

ISSN 0013-726X

\section{Corresponding author}

\section{Martin Faehndrich, MD}

Interventional Endoscopy

Department of Gastroenterology

Klinikum Dortmund

Beurhausstraße 40

44137 Dortmund

Germany

Fax: +49-231-95320510

martin.faehndrich@klinikumdo.de

martin.faehndrich@web.de 